\title{
EXTREME POINTS IN SPACES OF CONTINUOUS FUNCTIONS
}

\author{
V. I. BOGACHEV, J. F. MENA-JURADO, AND J. C. NAVARRO-PASCUAL
}

(Communicated by Dale Alspach)

\begin{abstract}
We study the $\lambda$-property for the space $\mathfrak{C}(T, X)$ of continuous and bounded functions from a topological space $T$ into a strictly convex Banach space $X$. We prove that the $\lambda$-property for $\mathfrak{C}(T, X)$ is equivalent to an extension property for continuous functions of the pair $(T, X)$. We show also that, when $X$ has even dimension, the $\lambda$-property is equivalent to the fact that the unit ball of $\mathfrak{C}(T, X)$ is the convex hull of its extreme points and that this last property is true if $X$ is infinite dimensional. As a result we get that the identity mapping on the unit ball of an infinite-dimensional strictly convex Banach space can be expressed as the average of four retractions of the unit ball onto the unit sphere.
\end{abstract}

Given a Banach space $X, B(X)$ denotes its closed unit ball, $S(X)$ the unit sphere of $X$, and ext $B(X)$ the set of extreme points of $B(X)$. Along this paper we will consider only real Banach spaces.

If $X$ is a Banach space whose unit ball has some extreme point, we define the $\lambda$-function of $X$ by

$$
\lambda(x)=\operatorname{Sup}\{\alpha \in[0,1]: \exists e \in \operatorname{ext} B(X) \text { and } y \in B(X) \text { with } x=\alpha e+(1-\alpha) y\}
$$

for every $x$ in $B(X)$.

$X$ is said to have the $\lambda$-property if $\lambda(x)>0$ for all $x$ in $B(X) . X$ is said to have the uniform $\lambda$-property if $X$ has the $\lambda$-property and, in addition, satisfies

$$
\operatorname{Inf}\{\lambda(x): x \in B(X)\}>0 .
$$

These concepts were introduced and studied for the first time by Aron and Lohman in [1]. It was proved later that $X$ has the $\lambda$-property if, and only if, $X$ has the convex series representation property (C.S.R.P.), that is, every point in its unit ball can be expressed as an infinite convex combination of extreme points of the unit ball [2].

For $T$ a topological space and $X$ a Banach space, $\mathfrak{C}(T, X)$ will stand for the space of continuous and bounded mappings from $T$ into $X$, endowed with its usual supremum-norm. It is easy to see that, if $e$ is an extreme point of the unit ball of $\mathfrak{C}(T, X)$, then $\|e(t)\|=1$ for all $t$ in $T$, but the converse is

Received by the editors August 11, 1992 and, in revised form, February 17, 1993.

1991 Mathematics Subject Classification. Primary 46B20; Secondary 46E15.

Key words and phrases. $\lambda$-property, uniform $\lambda$-property, extreme point, strictly convex space, covering dimension. 
not true in general. However, if we suppose that $X$ is a strictly convex Banach space, then $e$ is an extreme point of the unit ball of $\mathfrak{C}(T, X)$ if, and only if, $\|e(t)\|=1$, for all $t$ in $T$.

Our first goal in this paper is to study the $\lambda$-property in $\mathfrak{C}(T, X)$ when $X$ is a strictly convex Banach space. We need the following lemma which can easily be proved.

1. Lemma. Let $X$ be a Banach space whose unit ball has some extreme point. Then for every $x$ in $B(X)$

$$
\lambda(x)=\operatorname{Sup}\{\alpha \geq 0: \exists e \in \operatorname{Ext} B(X) \text { with } \alpha+\|x-\alpha e\| \leq 1\} .
$$

2. Theorem. Let $T$ be a topological space and $X$ a strictly convex Banach space. The following conditions are equivalent:

(i) $Y=\mathfrak{C}(T, X)$ has the uniform $\lambda$-property.

(ii) $Y$ has the $\lambda$-property.

(iii) Every continuous mapping $y: A \rightarrow S(X)$ defined on a closed set $A$ of $T$ such that there exists a continuous mapping $y_{0}: T \rightarrow B(X)$ satisfying $y_{0}(t)=$ $y(t)$ for $t$ in $A$ has a continuous extension $e$ from $T$ into $S(X)$.

Moreover, if $Y$ has the $\lambda$-property, then $\lambda(y)=(1+m(y)) / 2$ for every $y$ in $B(Y)$, where $m(y)=\operatorname{Inf}\{\|y(t)\|: t \in T\}$.

Proof. (i) $\Rightarrow$ (ii) This is obvious.

(ii) $\Rightarrow$ (iii) Let $A$ be a closed set in $T$ and $y: A \rightarrow S(X)$ a continuous mapping such that there exists $y_{0}: T \rightarrow B(X)$ continuous with $y_{0}(t)=y(t)$, for $t$ in $A$. Then $y_{0}=B(Y)$ and by (ii) there exist $e \in \operatorname{Ext} B(Y), z \in B(Y)$, and $0<\alpha \leq 1$ satisfying $y_{0}=\alpha e+(1-\alpha) z$, so, for every $t$ in $A$, we have $y(t)=\bar{y}_{0}(t)=\alpha e(t)+(1-\alpha) z(t)$. Since $y(t) \in S(X)$ and $X$ is strictly convex, we get $e(t)=y(t)$ for every $t$ in $A$ and $e: T \rightarrow S(X)$ is a continuous extension of $y$.

(iii) $\Rightarrow$ (i) We are going to calculate the $\lambda$-function for $Y$. Let $y$ be in $B(Y)$, $e \in \operatorname{Ext} B(Y)$, and $\alpha \geq 0$ such that $\alpha+\|y-\alpha e\| \leq 1$. Then for every $t$ in $T$ we have $\alpha+\|y(t)-\alpha e(t)\| \leq 1$; hence, $2 \alpha-\|y(t)\| \leq 1$, that is, $\alpha \leq(1+m(y)) / 2$. Now Lemma 1 gives us $\lambda(y) \leq(1+m(y)) / 2$. If $m(y)>0$, we can define $e(t)=y(t) /\|y(t)\|$ for each $t$ in $T$, and $e$ is an extreme point in $B(Y)$. Now for any $t$ in $T$ we have

$$
\begin{aligned}
& \frac{1+m(y)}{2}+\left\|y(t)-\frac{1+m(y)}{2} e(t)\right\| \\
& \quad=\frac{1+m(y)}{2}+\left|\|y(t)\|-\frac{1+m(y)}{2}\right| \\
& \quad=\frac{1+m(y)}{2}+\frac{1}{2}|\|y(t)\|-1+\|y(t)\|-m(y)| \\
& \leq \frac{1+m(y)}{2}+\frac{1}{2}(1-\|y(t)\|+\|y(t)\|-m(y))=1 .
\end{aligned}
$$

So $(1+m(y)) / 2+\|y-((1+m(y)) / 2) e\| \leq 1$ and, again, by Lemma $1, \lambda(y) \geq$ $(1+m(y)) / 2$. This together with the above inequality gives us the result in this case.

If $m(y)=0$, let $\alpha$ be a positive number with $\alpha<\frac{1}{2}$.

Let us write $A=\{t \in T:\|y(t)\| \geq 1-2 \alpha\}$. Then $A$ is a closed set in $T$. 
Now let us define $y_{0}: T \rightarrow B(X)$ by

$$
y_{0}(t)= \begin{cases}y(t) /\|y(t)\| & \text { if } t \in A \\ y(t) /(1-2 \alpha) & \text { if } t \notin A\end{cases}
$$

Then $y_{0}$ is a continuous mapping satisfying $y_{0}(t)=y(t) /\|y(t)\|$ for $t$ in $A$; by (iii) there exists a continuous mapping $e: T \rightarrow S(X)$ with $e(t)=y(t) /\|y(t)\|$ for every $t$ in $A$. So $e \in \operatorname{Ext} B(Y)$ and, if $t \in A$, we have

$$
\alpha+\|y(t)-\alpha e(t)\|=\alpha+\left\|y(t)-\alpha \frac{y(t)}{\|y(t)\|}\right\|=\alpha+|\|y(t)\|-\alpha| \leq 1 ;
$$

and if $t \notin A$, then

$$
\alpha+\|y(t)-\alpha e(t)\| \leq 2 \alpha+\|y(t)\|<2 \alpha+1-2 \alpha=1 .
$$

Hence $\alpha+\|y-\alpha e\| \leq 1$ and, by Lemma $1, \lambda(y) \geq \alpha$ for every $\alpha<\frac{1}{2}$, so $\lambda(y) \geq \frac{1}{2}$ and the proof is finished.

3. Remark. The above theorem is not true if one drops the condition of $X$ being strictly convex. If one considers $T=\mathbb{N} \cup\{\infty\}$, the one-point compactification of the natural numbers and $X=\mathbb{R}^{n}$ with any norm whose unit ball has a set of extreme points which is not closed, then $\mathfrak{C}(T, X)$ fail to have the $\lambda$-property and $X$ satisfies assertion (iii) in the theorem.

Taking into account that, when $X$ is an infinite-dimensional Banach space, the unit sphere of $X$ is a retract of the unit ball of $X$, we get that condition (iii) in the above theorem is always true if $X$ is infinite dimensional, so we have

4. Corollary. Let $X$ be an infinite-dimensional strictly convex space and $T a$ topological space. Then $Y=\mathfrak{C}(T, X)$ has the uniform $\lambda$-property and $\lambda(y)=$ $(1+m(y)) / 2$ for every $y$ in $B(Y)$.

When $X$ is finite-dimensional, condition (iii) is not always true (take $T=$ $B(X), A=S(X)$, and $y$ the identity mapping in $S(X))$ but, if $T$ is a completely regular space, it was proved by Smyrnov that the condition (iii) is equivalent to $\operatorname{dim}(X) \leq n-1\left[9\right.$, Theorem $\left.9_{t}\right]$, where $n$ is the algebraic dimension of $X$ and $\operatorname{dim}(T)$ is the covering dimension of $T$ (see [5] for definitions), so we get

5. Corollary. Let $X$ be an $n$-dimensional strictly convex space and $T$ a completely regular space. Then the following conditions are equivalent:

(i) $\mathfrak{C}(T, X)$ has the $\lambda$-property.

(ii) $\mathfrak{C}(T, X)$ has the uniform $\lambda$-property.

(iii) $\operatorname{dim}(T) \leq n-1$.

6. Remark. In the proof of the implication (iii) $\Rightarrow$ (i) of Theorem 2 we follow the proof by Aron and Lohman of [1, Theorem 1.6], which is our Corollary 4 in case $T$ is a metric compact space. In fact, by assuming only that $T$ is a compact Hausdorff space, Peck had proved before that, when $X$ is an infinitedimensional strictly convex Banach space, every element in the unit ball of $\mathfrak{C}(T, X)$ can be expressed as the average of four extreme points of the unit ball [8, Theorem 5]. When $X$ is not necessarily infinite-dimensional, Aron and Lohman proved [1, Theorem 1.9], which is a consequence of our Corollaries 4 and 5. 
If one considers $n=1$ in Corollary 5 together with [2, Theorem 1], one gets that $\mathfrak{C}(T, \mathbb{R})$, for $T$ a compact Hausdorff space, has the convex series representation property if, and only if, $T$ is zero dimensional, which is the main result of [7], although this result was proved for the first time by Peck [8, Theorem 4]. To finish this remark let us say that Cantwell proved in [3, Theorem 2] that, when $T$ is a normal space and $X$ is a Hilbert space of dimension $n \geq 2$, condition (iii) in Corollary 5 is equivalent to the fact that the unit ball of $\mathfrak{C}(T, X)$ is the convex hull of its extreme points. In view of this result and the aforementioned result by Peck in the infinite-dimensional case, one can suspect that condition (iii) in Theorem 2 is, in fact, equivalent to a stronger condition than the $\lambda$-property for $\mathfrak{C}(T, X)$, that is, that the unit ball of $\mathfrak{C}(T, X)$ is the convex hull of its extreme points. We almost obtain this by a convenient adaptation of the proof given by Peck when $T$ is a compact Hausdorff space and $X$ is an infinite-dimensional strictly convex Banach space.

In the sequel we will say that the pair $(T, X)$ has the extension property if $T$ is a topological space and $X$ is a strictly convex Banach space satisfying condition (iii) in Theorem 2.

7. Lemma. Let $(T, X)$ have the extension property. Then if $f$ is a continuous mapping $f: T \rightarrow X$, and $\varepsilon>0$, there exists a continuous mapping $g: T \rightarrow X$ such that $f(t)=g(t)$ if $\|f(t)\| \geq \varepsilon$ and $\|g(t)\|=\varepsilon$ if $\|f(t)\| \leq \varepsilon$.

Proof. Let us define $F: T \rightarrow B(X)$ by

$$
F(t)= \begin{cases}f(t) /\|f(t)\| & \text { if }\|f(t)\| \geq \varepsilon, \\ \frac{1}{\varepsilon} f(t) & \text { if }\|f(t)\| \leq \varepsilon .\end{cases}
$$

Then $F$ is continuous and $A=\{t \in T:\|f(t)\|=\varepsilon\}$ is a closed set in $T$. Since $(T, X)$ has the extension property, there exists a continuous mapping $f_{0}: T \rightarrow$ $S(X)$ such that $f_{0}(t)=\frac{1}{\varepsilon} f(t)$ for every $t$ in $A$. Let us define $g: T \rightarrow X$ by

$$
g(t)= \begin{cases}f(t) & \text { if }\|f(t)\| \geq \varepsilon \\ \varepsilon f_{0}(t) & \text { if }\|f(t)\| \leq \varepsilon .\end{cases}
$$

Then $g$ is continuous and satisfies the conditions for which we are looking.

The above lemma was proved in [8, Corollary to Proposition 2] provided that $T$ is compact Hausdorff and $X$ is an infinite-dimensional Banach space.

Now we can prove the following corollary by using the above lemma in the same way it was proved by Peck (see the proof of Theorem 3 in [8]).

8. Corollary. Let $(T, X)$ have the extension property. Then every element in the unit ball of $\mathfrak{C}(T, X)$ can be expressed as the average of two elements in the unit ball of $\mathfrak{C}(T, X)$ which omit the origin.

In order to prove that the unit ball of $\mathfrak{C}(T, X)$ is the convex hull of its extreme points we must impose that $(T, X)$ has the extension property due to Theorem 2 and, in view of the above corollary, all we need to prove is that every element of the unit ball omitting the origin can be expressed as a convex combination of extreme points. We prove this with an additional condition on $X$ in the next Proposition, in whose proof we follow the proof of [8, Theorem 5]. 
9. Proposition. Let $X$ be a strictly convex space such that there exists a continuous mapping $v: S(X) \rightarrow S(X)$ with $v(x) \neq x$ and $v(x) \neq-x$ for every $x$ in $S(X)$. Then there exist continuous mappings $\phi_{1}, \phi_{2}: B(X)-\{0\} \rightarrow S(X)$ such that $x=\frac{1}{2}\left(\phi_{1}(x)+\phi_{2}(x)\right)$ for every $x$ in $B(X)-\{0\}$.

Proof. Let us denote by $r$ the mapping from $B(X)-\{0\}$ into $S(X)$ defined by $r(x)=x /\|x\|$. We define $\Gamma:[0,2] \times(B(X)-\{0\}) \rightarrow S(X)$ by

$$
\Gamma(t, x)= \begin{cases}r((1-t) r(x)+t v(r(x))) & \text { if } 0 \leq t \leq 1, \\ r((2-t) v(r(x))-(t-1) r(x)) & \text { if } 1 \leq t \leq 2 .\end{cases}
$$

Then $\Gamma$ is continuous, and if we fix $x$ in $B(X)-\{0\}$, we have

$$
\begin{aligned}
& \|2 x-\Gamma(0, x)\|=\|2 x-r(x)\|=|2\|x\|-1| \leq 1, \\
& \|2 x-\Gamma(2, x)\|=\|2 x+r(x)\|=2\|x\|+1>1,
\end{aligned}
$$

so there is some $t$ in $[0,2]$ such that $\|2 x-\Gamma(t, x)\|=1$. Now by [8, Lemma $3]$ there is only one $t$ for which the above equality holds; if we denote it by $t(x)$, we are going to prove that the mapping $x \rightarrow t(x)$ is continuous. If $x \in B(X)-\{0\}$ is a point of discontinuity of the above mapping, we can find a sequence $\left\{x_{n}\right\}$ of elements in $B(X)-\{0\}$ and $t$ in $[0,2]$ such that $\left\{x_{n}\right\} \rightarrow x$ and $\left\{t\left(x_{n}\right)\right\} \rightarrow t \neq t(x)$. Now the continuity of $\Gamma$ gives

$$
\left\{\left\|2 x_{n}-\Gamma\left(t\left(x_{n}\right), x_{n}\right)\right\|\right\} \rightarrow\|2 x-\Gamma(t, x)\|,
$$

so $\|2 x-\Gamma(t, x)\|=1$, and this contradicts the unicity of $t(x)$. So we can define $\phi_{1}(x)=\Gamma(t(x), x)$ and $\phi_{2}(x)=2 x-\phi_{1}(x)$ which are continuous mappings from $B(X)-\{0\}$ into $S(X)$ satisfying

$$
x=\frac{1}{2}\left(\phi_{1}(x)+\phi_{2}(x)\right) \text { for every } x \text { in } B(X)-\{0\} .
$$

From Corollary 8 and Proposition 9 we obtain

10. Theorem. Let $T$ be a topological space and $X$ a strictly convex space satisfying.

(a) $(T, X)$ has the extension property.

(b) There exists a continuous mapping $v: S(X) \rightarrow S(X)$ such that $v(x) \neq x$ and $v(x) \neq-x$ for every $x$ in $S(X)$.

Then $B(Y)=\operatorname{co}(\operatorname{Ext} B(Y))$, where $Y=\mathfrak{C}(T, X)$.

In fact, we have proved that, with the conditions in the above theorem, every element in the unit ball of $\mathfrak{C}(T, X)$ can be expressed as the average of four extreme points.

When $X$ is finite dimensional, it is known that $X$ satisfies condition (b) in the above theorem if, and only if, $X$ has even dimension, so we have

11. Corollary. Let $T$ be a completely regular space and $X$ a strictly convex space with dimension equal to $2 n$ with $n \in \mathbb{N}$. The following conditions are equivalent:

(i) $Y=\mathfrak{C}(T, X)$ has the $\lambda$-property.

(ii) $Y$ has the uniform $\lambda$-property.

(iii) $B(Y)=\operatorname{co}(\operatorname{Ext} B(Y))$.

(iv) $\operatorname{dim}(T) \leq 2 n-1$. 
Cantwell proved the equivalence between (iii) and (iv) in the above result without restrictions on the dimension of $X$, but he assumes that $X$ is a Hilbert space and $T$ is a normal space [3, Theorem II]. In fact, in our corollary and in the quoted theorem by Cantwell, one can suppose that $T$ is compact by using [5, Theorem 3.6.1]. This result by Cantwell says that condition (b) in Theorem 10 is no longer necessary to get that the unit ball of $\mathfrak{C}(T, X)$ is the convex hull of its extreme points.

If $X$ is infinite dimensional, condition (a) in Theorem 10 is satisfied. Condition (b) is obviously satisfied in Banach spaces which admit complex structure, but this is not always the case (see, for example, [10] for information about this topic). Nevertheless, the next result shows that condition (b) is also true in the infinite dimensional case.

12. Proposition. Let $X$ be an infinite dimensional Banach space. Then there exists a continuous mapping $v: S(X) \rightarrow S(X)$ such that $v(x) \neq x$ and $v(x) \neq$ $-x$ for every $x$ in $S(X)$.

Proof. First suppose $X$ is separable. Then by [6, Proposition 1.f.3] there exist $\left\{x_{n}\right\}_{n \in \mathbb{Z}}$ a sequence in $S(X)$ and $\left\{f_{n}\right\}_{n \in \mathbb{Z}}$ a sequence in $X^{*}$ verifying $f_{n}\left(x_{m}\right)=$ $\delta_{n m}$ for all $n, m$ in $\mathbb{Z}$ and $\left\{f_{n}\right\}_{n \in \mathbb{Z}}$ separates points in $X$. Let us define

$$
A(x)=\sum_{n=-\infty}^{+\infty} \alpha_{n} f_{n+1}(x) x_{n} \quad(x \in X)
$$

where $\alpha_{0}=1$ and $\alpha_{n}=|n|^{-|n|}\left(1+\left\|f_{n+1}\right\|\right)^{-1} \quad(n \in \mathbb{Z}-\{0\})$. Then $A$ is a compact operator in $X$. If $A(x)=0$, then $f_{n}(x)=0$ for all $n$ in $\mathbb{Z}$ and so $x=0$. If $A(x)=\lambda x$ for some scalar $\lambda \neq 0$ and $x$ in $S(X)$ then $\alpha_{n} f_{n+1}(x)=\lambda f_{n}(x)$ for all $n$ in $\mathbb{Z}$. Hence

$$
f_{n+1}(x)=n^{n} \lambda^{n} f_{1}(x)\left(1+\left\|f_{n+1}\right\|\right) \prod_{k=1}^{n-1} k^{k}\left(1+\left\|f_{k+1}\right\|\right)
$$

for all $n$ in $\mathbb{N}$ and $\left|f_{n+1}(x)\right|>1+\left\|f_{n+1}\right\|$ for some $n$ big enough, and this is a contradiction.

So if we define $v: S(X) \rightarrow S(X)$ by $v(x)=A(x) /\|A(x)\|$ then $v(x) \neq x$ and $v(x) \neq-x$ for every $x$ in $S(X)$ and $v$ is continuous.

If $X$ is not separable, let $X_{0}$ be an infinite-dimensional separable closed subspace of $X$. Then by [4, Theorem 6.2] there is $F: S(X) \rightarrow S\left(X_{0}\right)$ continuous such that $F(x)=x$ for every $x$ in $S\left(X_{0}\right)$. By the first part of the proof there is $v_{0}: S\left(X_{0}\right) \rightarrow S\left(X_{0}\right)$ continuous and $v_{0}(x) \neq x, v_{0}(x) \neq-x$ for every $x$ in $S\left(X_{0}\right)$. Then $v=v_{0} F: S(X) \rightarrow S(X)$ is continuous and $v(x) \neq x, v(x) \neq-x$ for every $x$ in $S(X)$.

Taking into account the commentaries we have made before we obtain

13. Corollary. Let $T$ be a topological space and $X$ an infinite-dimensional strictly convex Banach space. Then $B(Y)=\operatorname{co}(\operatorname{Ext} B(Y))$, where $Y=\mathfrak{C}(T, X)$.

As we have already said the above corollary was proved by Peck [8, Theorem 5] by assuming that $T$ is compact Hausdorff. Our corollary allows us to get the following striking result which can not be derived from the result by Peck. 
14. Corollary. Let $X$ be an infinite-dimensional strictly convex Banach space. Then there exist $e_{1}, e_{2}, e_{3}, e_{4}$ retractions of the unit ball of $X$ onto the unit sphere of $X$ such that

$$
x=\frac{1}{4}\left(e_{1}(x)+e_{2}(x)+e_{3}(x)+e_{4}(x)\right)
$$

for every $x$ in $B(X)$.

\section{REFERENCES}

1. R. M. Aron and R. H. Lohman, A geometric function determined by extreme points of the unit ball of a normed space, Pacific J. Math. 127 (1987), 209-231.

2. R. M. Aron, R. H. Lohman, and A. Suarez, Rotundity, the C.S.R.P., and the $\lambda$-property in Banach spaces, Proc. Amer. Math. Soc. 111 (1991), 151-155.

3. J. Cantwell, A topological approach to extreme points in function spaces, Proc. Amer. Math. Soc. 19 (1968), 821-825.

4. J. Dugundji, An extension of Tietze's Theorem, Pacific J. Math. 1 (1951), 353-367.

5. R. Engelking, General topology, Heldermann Verlag, Berlin, 1989.

6. J. Lindenstrauss and L. Tzafriri, Classical Banach spaces. I, Springer-Verlag, Berlin, 1977.

7. D. Oates, A sequentially convex hull, Bull. London Math. Soc. 22 (1990), 467-468.

8. N. T. Peck, Extreme points and dimension theory, Pacific J. Math. 25 (1968), 341-351.

9. Yu. M. Smirnov, On the dimension of proximity spaces, Mat. Sb. 38 (1956), 283-302; English transl., Amer. Math. Soc. Transl. Ser. 2, vol. 21, Amer. Math. Soc., Providence, RI, 1962, pp. 1-20.

10. S. J. Szarek, A superreflexive Banach space which does not admit complex structure, Proc. Amer. Math. Soc. 97 (1986), 437-444.

Department of Mechanics and Mathematics, Moscow State University, Leninskie GORY, MOSCOW, RUSSIA

Departamento de Análisis Matemático, facultad de Ciencias, Universidad de Granada, 18071-Granada, Spain

E-mail address: jfmena@ugr.es

Departamento de Análisis Matemático, Facultad de Ciencias Experimentales de Almeria, Universidad de Granada, 04071-Almeria, Spain 